\title{
The Effect of Busulfan on Body Weight, Testis Weight and MDA Enzymes in Male Rats
}

\author{
Nasibeh Hosseini Ahar ${ }^{1}$, Arash Khaki ${ }^{*}$,Ghasem Akbari ${ }^{3}$, Marefat Ghaffari Novin ${ }^{4}$
}

\begin{abstract}
Objectives: Busulfan is one of the anti-cancer drugs, despite of the tremendous usefulness in biotechnology and therapy of chronic diseases such as leukemia, lymphoma, ovarian cancer and it can lead to impaired spermatogenesis. The aim of this study was to seen body and testicular weight and serum Malondialdehyde (MDA) enzyme in rat was performed following the use of busulfan.

Material and Methods: This study was conducted on 20 adult male rats ranging in age from 6 to 8 weeks, Animals in the two group includes a control group and the experimental group that received $50 \mathrm{mg} / \mathrm{kg}$ busulfan as a single intra peritoneal injection and after 8 weeks, body and testis weight and serum MDA levels were measured. Then Data were analyzed with SPSS 16 . P $<0.05$ was considered significant, and results were compared between the two groups.

Results: single dose of busulfan, induced its effects on body, testis weights and serum MDA levels. Body weight in experimental and control groups, was respectively, $297.40 \mathrm{gr}$ and $301.00 \mathrm{gr}$, Body weight of rats in the experimental group than the control group was decreased, but the difference was not significant $(\mathrm{P}>0.05)$. Testicular weight in both control and experimental groups, respectively, were $1.45 \mathrm{gr}$ and gr 0.960 . Difference between the two groups was significant decreased $(\mathrm{P}>0.05)$. Comparison of serum MDA of control and experimental groups, were showed $3.81 \pm 1.5$ and $6.9 \pm 1.1 \mathrm{nmol} /$ lit. No difference between the two groups was significant increased $(\mathrm{P}>0.05)$.

Conclusion: It can be concluded that the use of busulfan can reduced body weight and testicular weight, and increased serum MDA and could be side effects in reproduction process.
\end{abstract}

Keywords: Busulfan, Serum MDA, Testis Weight, Body Weight

\section{Introduction}

Several factors have effect on very sensitive and complex process named spermatogenesis, and Lead to infertility or reduced fertility (1). One of the most important factors affecting spermatogenesis is chemotherapy that by creating adverse effects on the cell division process will eventually lead toazoospermia (2). Busulfan is alkylating chemotherapeutic agents that used in order to treat chronic leukemia, ovarian cancer, lymphoma and Milo Proliferative Disorders and also used before bone marrow transplantation in cancer patients (3). It is in a class of medications called alkylating agents.

This drug with non-specific binding to DNA strand, acts on the cell cycle and inhibit DNA activity. In fact, its cytotoxic effect is by interfering in DNA replication and RNA transcription (4). After one or two intraperitoneal injection, it can destroy high amount of spermatogenesis (5).

Busulfan has side effects on various organs including the liver, skin, bladder, nervous system and gonadal function, and is potentially carcinogenic and mutagenic (6). Despite of the tremendous usefulness in biotechnology and therapy of chronic diseases, its exact effects on the testis structure and parameters of epididymal-spermatozoa has not yet been well studied. Thus, the present study examined body weight, testicular weight and serum MDA levels in rats was performed following the use of busulfan.

\begin{abstract}
Material and Methods
Animals

Twenty Wistar male rats ranging in age from 6 to 8 weeks $(200 \pm 20 \mathrm{~g})$ were used. Animals Were randomly allocated into two groups, control group $(n=10)$ and experimental group $(n=10)$ that received a single intraperitoneal injection of busulfan (50 mg/kg body weight) diluted in sesame oil as previously described (19.20). animals were housed in wire cages at $22 \pm 1{ }^{\circ} \mathrm{C}$ under a 12 -h lightdark cycle with $70 \%$ humidity and fed a standard diet and water. Animals were maintained and experiments were conducted in accordance with the Principles of Laboratory Animal Care of Tabriz University of Medical Sciences, Iran.
\end{abstract}

\section{Drugs preparation}

Preparation of busulfan solution: Busulfan (Sigma, USA) was first dissolved in DMSO (dimethyl sulfoxide), (Sigma, 
USA), then an equal volume of sterile water was added to obtain a final busulfan concentration of $5 \mathrm{mg} / \mathrm{ml}$.

\section{Surgical procedure}

At the end of the treatment period, (56th day) the rats were weighed and anesthetized by using intraperitoneal Pentobarbital sodium (40 mg/kg), then killed; the peritoneal cavity was opened through a lower transverse abdominal incision. As well as, testes in control and experimental groups were immediately removed and fixed by perfusion with $4 \%$ formaldehyde in buffered solution for 20 minutes and after wards their left testes were taken out and weighed. (A\&D GF600, Germany)

\section{Measurement of MDA}

Before perfusion, in order to measure the plasma MDA levels, blood samples were immediately collected by cardiac puncture and the plasma separated from the blood cells by centrifugation ( $2500 \mathrm{rpm}$ for $30 \mathrm{~min}$ ). All blood samples were then immediately stored at $-20{ }^{\circ} \mathrm{C}$ until further analyses. MDA levels were determined with TBA assay, according to the method as described by Rao et al. (7).

\section{Data analysis}

All experimental data are presented as means \pm SD. Each experiment was performed at least three times and subjected to statistical analysis. Representative experiments are presented in the Figure. For statistical analysis, analysis of variance (Mann-Whitney) was performed to determine whether there were differences among two groups $(\mathrm{P}<0.05)$. A $\mathrm{P}$ value less than 0.05 was considered significant.

\section{Results}

\section{Body and testis weight}

The obtained results in this study were illustrated in Table 1. There was no significant difference in body weights between two groups $(\mathrm{P}<0.69)$. Testis weight was reduced significantly in experimental group in comparison with the control group $(\mathrm{P}<0.008$; Figures 1and 2$)$.

\section{MDA Assay}

MDA levels of plasma of rats in experimental group were significantly higher than in control group $(\mathrm{P}<0.032$; Table 1; Figure 3).

\section{Discussion}

The administration of busulfan to male patients with malignant diseases may cause temporary or permanent sterility (8). Estimation of testis parameters such as body and testis weight and MDA levels of plasma by stereological methods lead to better evaluation of the spermatogenesis process.

In the present study, we have determined the extent of changes in mouse testis weight, body weight and MDA levels following Busulfan administration. Male rats were subdivided into two groups, control and experimental

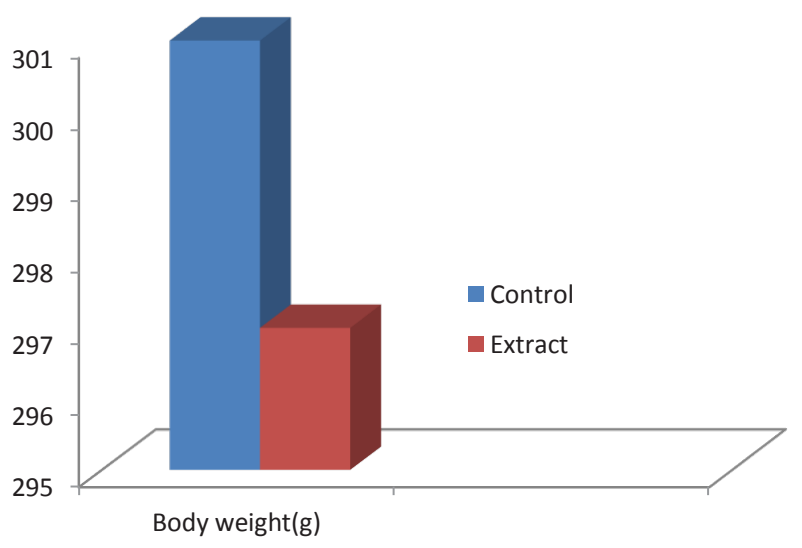

Figure 1. Body weight of control and experimental groups

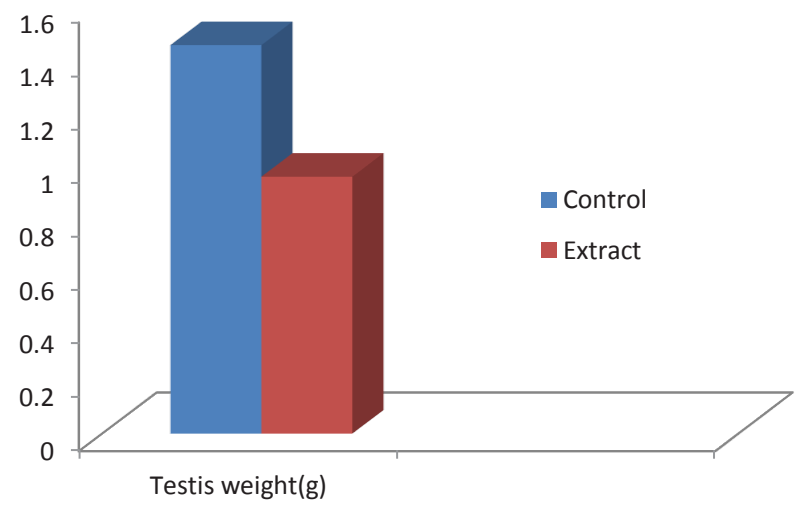

Figure 2. Testis weight of control and experimental groups

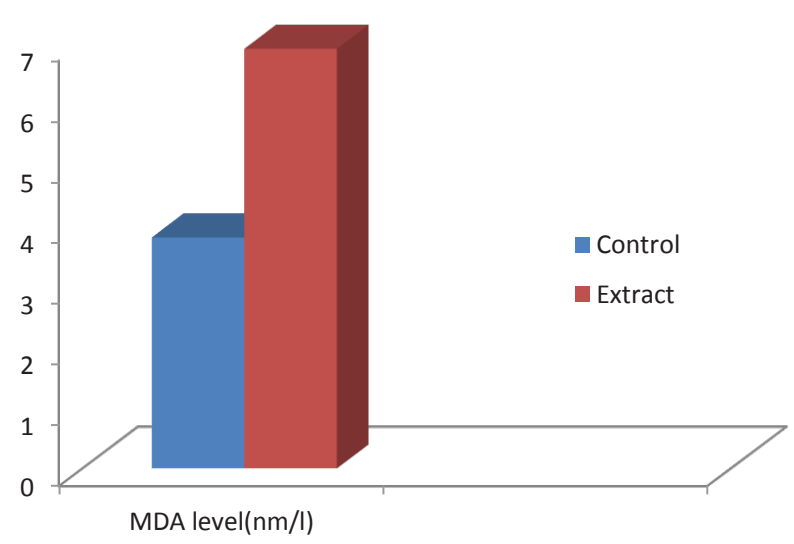

Figure 3. MDA levels of control and experimental groups

group. The chosen period of time ( 6 and 8 weeks) provided sufficient time to monitor the changes of spermatogenesis factor in testes in busulfan treated animals. Also, according to Nagano et al. (9) experimental group receiving Busulfan showed decreased in body weight, testicular weight and increased adjusting blood MDA enzyme in comparison to control group. This result could be due to cytotoxic effects of Busulfan with Transferring the alkyl group(s) to various 
Table 1. Body weight, Testis weight and MDA levels of control and experimental groups.

\begin{tabular}{|c|c|c|c|c|}
\hline \multirow[b]{2}{*}{ Groups } & \multirow[t]{2}{*}{$\mathrm{N}$} & \multicolumn{3}{|c|}{ Variables } \\
\hline & & Body weight (g) & Testis weight (g) & MDA level $(\mathrm{nm} / \mathrm{l})$ \\
\hline Control & 5 & $301 \pm 22.47$ & $1.45 \pm 0.1$ & $3.81 \pm 1.5$ \\
\hline Experimental & 5 & $297.4 \pm 7.98$ & $0.96 \pm 0.07 *$ & $6.9 \pm 1.1^{*}$ \\
\hline
\end{tabular}

Data are presented as mean \pm SE.

*Significant different at $\mathrm{P}<0.05$ level (compared with the control group).

cellular. However, DNA alkylation events may constitute major incitements leading to cell death (10). There are several types of chemotherapeutic drugs which show cell damage stage-specific cytotoxicity to spermatogonia (11). Busulfan partially eliminates stem cells because of its alkylating nature (12) and kills cells by producing free radicals (13). Therefore, it seems busulfan inhibit the spermatogenesis process, especially by oxidative damage. Other mechanism suggested that busulfan increased the level of ck18, a surface marker on sertoli cell. The elevation of this marker caused spermatogenesis disorder and infertility (14). In this study, busulfan decreases body weight and testes weight. Zheng Wei et al. showed that there was a direct relationship between testis weight and germinal cells number in primates (15). Bucci et al. showed that busulfan caused chromosomal abnormalities and dominant lethal mutations in sperm (16).

This study also showed that busulfan increased MDA levels of plasma. Malondialdehyde can be used as a marker of oxidative stress and a potential marker for predicting assisted reproductive techniques (ART) outcomes (17).

Hosseinzadeh Colagar et al. in 2013 observed MDA concentration in oligozoospermic and azoospermic men was significantly higher than normozoospermic. And represented evaluation of plasma MDA could be beneficial diagnostic tool for defining sperm fertilization potential (18). Therefore, it is suggested that, these parameters (MDA level, body weight, and testis weight) could help in distinction and treatment of male infertility especially in idiopathic cases.

\section{Conclusion}

The present study provides detailed information on the effects of busulfan on testis weight, body weight and MDA levels. The information has the potential to modify and improve the administration of busulfan to increase its efficiency to make a reliable infertile recipient animal for Future studies, in one hand, and also to decrease its numerous side-effects in the clinical application of the drug, on the other hand.

\section{Ethical issues}

The local ethics committee approved the study.

\section{Conflict of interests}

Authors declare that there is no any conflict of interests.

\section{Acknowledgments}

This research was supported by Women's Reproductive Health Research Center, Tabriz University of Medical
Sciences, Tabriz, Iran. The code of this research protocol was 9217.

\section{References}

1. Johari H, Sharifi E, Ansari N, Hosseini M, Amiri F. Effect of Hydro Alcoholic Ginger Extracts on the Body Weight, Testis Weight and Spermatogenesis in Male Rats Undergoing Chemotherapy with Cyclophosphamide.The Journal of ShahidSadoughi University of Medical Sciences 2010;17(5): 365-374.

2. Anderson PHO, Knoben JE, Troutman WG. Hand book of clinical data. 10th edition. Lonon:MC Graw Hill;2002.

3. Anjamrooz SH, Movahedin M, Mowla SJ, Pour Bairanvand $\mathrm{SH}$. Assessment of morphological and functional changes in the mouse testis and Epididymal sperms following Busulfan treatment, Iranian Biomedical Journal 2007;11(1):15-22.

4. Baazm M, Darabi MR, Babaei S, Tabeli R. Improvement in sperm parametres after nsing gonadotropin- releasing hormone analogue in busulfan treated prepubertal mice, Arak Medical University Journal 2013;16(79):11-18.

5. Change YJ, Lee WK, Kim HG. Oxidative stress in rat model of preeclampsia and clinical correlates. Korean J Physiol Pharmacol 2007; 11(3):129-133.

6. Chi H, Chun K, Son H, Kim J, Kim G, Roh S. Effect of genistein administration on the recovery of spermatogenesis in the busulfan- treated rat testis, Clin Exp Reprod Med 2013; 40(2): 60-66.

7. Rao B, Soufir JC, Martin M, David G. Lipid peroxidation in human spermatozoa as related to midpiece abnormalities and mortility. Gamete Res 1989;24(2): 127-134.

8. Howell SJ, Shalet SM. Spermatogenesis after cancer treatment : damage and recovery. J Natl Cancer Inst Monogr 2005;5(34): 12-17.

9. Nagano M, Avarbock MR, Brinster RL. Pattern and kinetics of mouse donor spermatogonial stem cell colonization in recipient testes. Biol Reprod 1999; 60(8):1429-1436.

10. Molenaar RD, Rooij DG, Rommerts FF, Reuvers PJ, Vander Melen HJ. Specific destruction of Lydig cells on mature rats after invivo administration of ethane dimethyl sulfonate. Biol Reprod 1985;33: 1213-1222.

11. Nudell DM, Monoski MM, Hipshultz LI. Common medications and drugs: how they affect male fertility. Urol Clin North AM 2002; 29(4): 965-973.

12. Ray S. Evalutions of antiperoxidative potential of ascorbic acid on busulfan- induced lipid peroxidation 
using 4-hydroxy- 2- non ental and nitric oxide as model markers. International Journal of Pharmtech Resaerch 2012; 3(3): 896-900.

13. Ray A. Evalution of protective role of morin on Busulfan- induced lipid peroxidation. International Journal of Pharmtech Resaerch 2011;3(4): 2222-7.

14. Ray S. Exploring protective role of Ascorbic acid on Busulfan- induced lipid peroxidation. Research Journal of Pharmaceutical, Biological and Chemical Sciences 2011; 2(4): 702-708.

15. Zheng wei Y, McLachlan RI, Bremner WJ, Wreford NG. Quantitative (stereological) study of the normal spermatogenesis in the adult monkey (Macacafascicularis).J Androl 1997; 18: 681-687.

16. Bucci LR, Meistrich ML. Effects of busulfan on murine spermatogenesis: cytotoxicity, sterility, sperm abnormalities, and dominant lethal mutations. Mutat Res 1987; 176: 259-268.

17. Hosseinzadeh Colagar A, Karimi F, Jorsaraei SG. Correlation of sperm parameters with semen lipid peroxidation and total antioxidants levels in asthenoand oligoasheno- teratospermic men. Iran Red Crescent Med J 2013;15(9):780-5.

Copyright ( 2014 The Author(s); This is an open-access article distributed under the terms of the Creative Commons Attribution License (http://creativecommons.org/licenses/by/4.0), which permits unrestricted use, distribution, and reproduction in any medium, provided the original work is properly cited. 